\title{
KSHV Inflammatory Cytokine Syndrome
}

National Cancer Institute

\section{Source}

National Cancer Institute. KSHV Inflammatory Cytokine Syndrome. NCI Thesaurus. Code C125711.

A syndrome caused by Kaposi sarcoma-associated herpesvirus (KSHV) infection. It manifests with fever, weight loss, and fluid retention in the legs or abdomen. Patients are at risk of developing KSHV-related cancers including Kaposi sarcoma and lymphoma. 